\title{
A mathematical model for the starting process of discharge lamps
}

\author{
Shigeaki WADA* and Koichiro TANIGAWA*
}

\begin{abstract}
Several mathematical models for discharge lamps have already been offered. However, all these models are only used for the operating state. There are not any models for the starting process.

This paper is concerned with the mathematical model for the starting process of discharge lamps. Newly introducing the concept of heat quantity, the authors were able to produce a mathematical model to represent the starting process of the low pressure sodium lamp.

In this paper, the following comparison between the theoretical results using this model and experimental results on the low pressure sodium lamp are discussed.
\end{abstract}

(1) Lamp voltage and current waveforms in the starting process.

(2) Evaluation whether or not the lamp can get to operating state.

These investigations showed that this model is available for representing the starting process of the low pressure sodium lamp.

\section{Introduction}

Many semiconductor starting devices and operating devices for discharge lamps have been developed. Some of them have already been practically used. It is supposed that they will be more and more a important research subjects. The necessity of mathematical research into the electrical characteristics of discharge lamps will be further increasing, along with this tendency towards efficiency and quality improvement in this research.

Hitherto, many attempts to represent the electrical characteristics of discharge lamps by differential equations have been made by V.J. Francis $^{1)}$, S. C. Peek and D. E. Spencer ${ }^{2)}$, Amano ${ }^{3)}$, Nishioka and Morimoto ${ }^{4}$, Masumi and Bou ${ }^{5)}$ (8). But, all these mathematical models represent only the operating state of discharge lamps (to be called an operating state) in a stationary state. There are no mathematical models which represent the starting process of discharge lamps (to be called a starting process) in the transitional state ranging from a power-on state to an operating state.

On the other hand, we, the authors, investigated a switch in parallel with a lamps, as shown in Fig. 1, as a semiconductor starting device for a low pressure sodium lamp and examined the method for leading a lamp to an operating state by switching on and off. As a result, when the number of times of switching on and off, and

* Research and Development Laboratory Matsushita Electric Works, LTD.

These works were presented partly at Conf. on Illum. Engng. Inst. of Japan in Tokyo, April 1979, and published in J. Illum. Engng. Inst. Jpn. 65, 10 (1981).

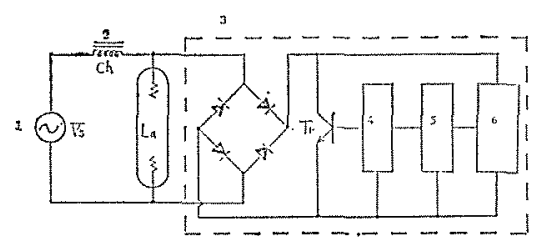

Fig. 1 Operating circuit for low pressure sodium lamp.

1. Power source

2. Ballast

3. Semiconductor startng device

4. Amplification circuit

5. Oscillation circuit

6. Detection circuit of lamp voltage

on-off ratio were changed, it became clear that a great difference in starting performance occurred.

We, the authors, carried on studies purposing the analytic treatment of this relationship. By a technological method, we developed ${ }^{9)-11)}$ a mathematical model enabling us to express a starting process by introducing first-order lag heat-quantity system into the methods of Nishioka and Morimoto $^{4}$.

Now, we calculate lamp voltage and current waveforms in the starting process, and evaluate whether or not the lamp can get to the operating state. In this paper, it is shown that the calculated results compared with experimental ones and discussed.

We consider these results are a help to the mathematical research of the operating state and starting process of discharge lamps in the future.

\section{Mathematical model for the starting process and lamp voltage - current waveforms}

There is the mathematical model proposed by 

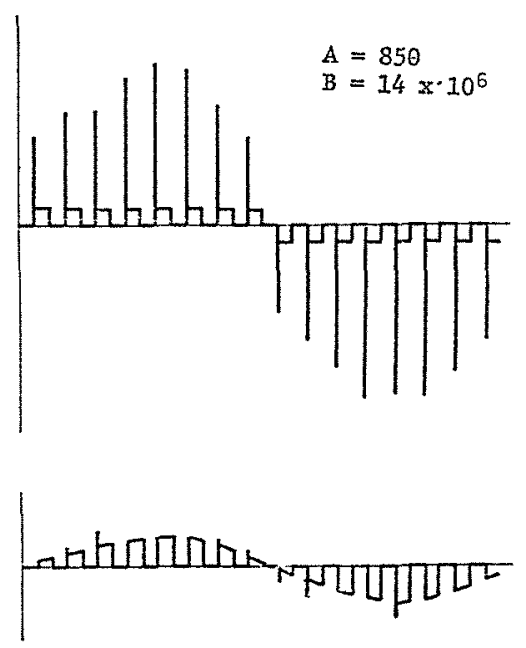

Fig. 2 Starting process waveforms by equation of Nishioka and Morimoto.

Nishioka and Morimoto ${ }^{4)}$ and Masumi and $\mathrm{Bou}^{5) ~ 8)}$ concurred that its accuracy is high even in cases where current off time exists.

$$
\frac{d G}{d t}=A i^{2}-B G^{2}
$$

$G$ means instantaneous conductance of lamps (S), $i$ is instantaneous value of lamp current (A), $d G / d t$ is time rate of change of conductance $(\mathrm{S} / \mathrm{s})$, $A\left(\mathrm{~S} /\left(\mathrm{s} \cdot \mathrm{A}^{2}\right)\right), B((1 / \mathrm{S} \cdot \mathrm{s}))$ are constants.

As equation (1) was originally made for the operating state, it cannot represent the starting process. Actually, even though the reproduction of the starting process is attempted, lamp voltage immediately decreases and lamp current immediately increases. It is translated into the operating state without passing the starting process as shown in Fig. 2.

On the other hand, authors, "with the assumption that the coefficient $A$ of the equation (1) is the function of first-order lag heat quantity against the electric power of the lamp", obtained the mathematical model representing the starting process and published this ${ }^{9)-11)}$.

\subsection{Mathematical model for the starting process}

Details are further explained as follows: Take a $35 \mathrm{~W}$ low pressure sodium lamp as an example, and consider the case that the coefficient $B$ in the equation (1) is fixed. Conductance $G$, the time rate of change $d G / d t$ of conductance, the lamp electric power $p_{L}$, coefficient $A$ are obtained from the lamp voltage $v_{L}$ waveforms and lamp current $i_{L}$ waveforms in the operating state. These relationships are shown in Fig. 3. In Fig. 3, as the lamp electric power $p_{L}$ increases, so does the coefficient $A$, and as the lamp electric power $p_{L}$ decreases, so does the coefficient $A$. Furthermore, the summit of coefficient $A$ has a little time lag compared to that of lamp electric power $p_{L}$.
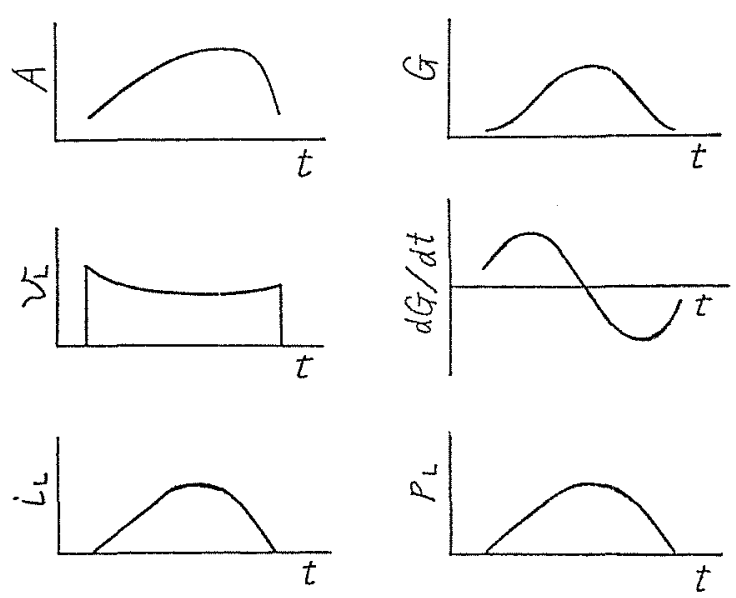

Fig. 3 Relationship between various electric quantities of lamp and coefficient $A$.

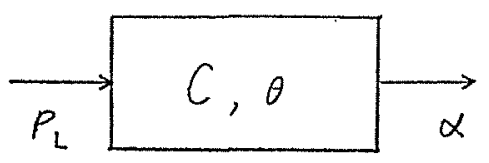

Fig. 4 First-order lag temperature system.

Then it was assumed that "coefficient $A$ was the function of the first-order lag temperature against lamp electric power $p_{L}$. Figure 4 shows the firstorder lag temperature system.

Where, $p_{L}$ is lamp electric power (W) which is input, $C$ is heat capacity $\left(\mathrm{J} /{ }^{\circ} \mathrm{C}\right), \alpha$ is heat emissivity* $\left(\mathrm{W} /{ }^{\circ} \mathrm{C}\right), \theta$ is temperature $\left({ }^{\circ} \mathrm{C}\right)$. Furthermore, variables are converted so that the system may be represented only by the information obtained from the lamp electric power $p_{L}$ and operating wave-forms. The temperature $\theta$ is replaced by an equation, heat quantity $Q=C \cdot \theta(J)$. It is assumed that coefficient $A$ is the function $A(Q)$ of the first-order lag heat quantity $Q$. The first-order lag heat quantity system is represented as follows:

$$
\frac{d Q}{d t}=p_{L}-\frac{Q}{T_{c}}
$$

Where, $Q$ is heat quantity $(J), d Q / d t$ is the time rate of change of heat quantity (W), $T_{c}=C / \alpha$ is time constant (s).

Next, the relationship between $A(Q)$ and heat quantity $Q$ is required. Presume that the time constant $T$. by comparing coefficient $A$ shown in Fig. 3 with the response of heat quantity $Q$. Where the time constant $T_{c}$ is parameter in the equation (2). By comparing the response of heat quantity $Q$ against this time constant $T_{c}$ with the coefficient $A$ in Fig. 3 in each time, the dotted line in Fig. 5 is obtained. $A(Q)$ is the double-valued function of heat quantity $Q$. Approximate this characteristic with the single-valued function shown by the unbroken line in Fig. 5. Then, $A(Q)$ is represented as follows:

$$
A(Q)=A_{1}\left(1-\exp \left(-Q / A_{2}\right)\right)
$$




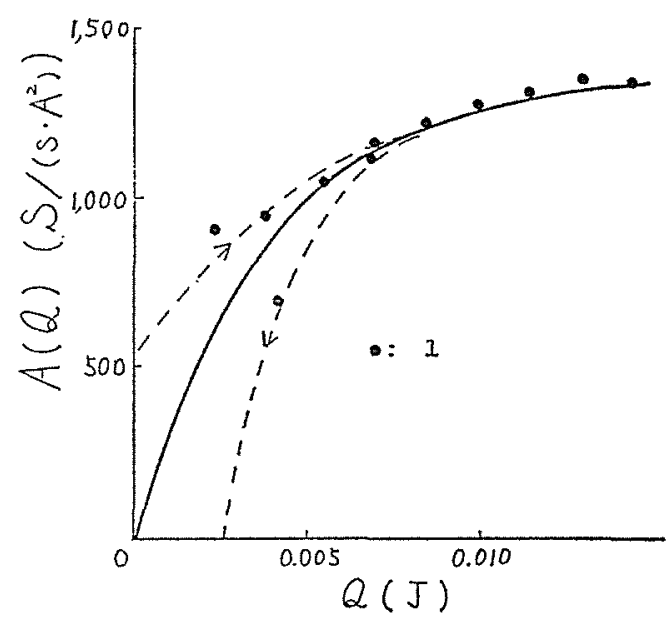

Fig. 5 Relationship between $A(Q)$ and heat quantity $Q$.

1. Actual measuring point

Where, $A_{1}\left(\mathrm{~S} /\left(\mathrm{s} \cdot \mathrm{A}^{2}\right)\right), A_{2}(\mathrm{~J})$ are constants.

Then, the time rate of change of the lamp conductance is represented as follows:

$$
\frac{d G}{d t}=A(Q) i^{2}-B G^{2}
$$

Make the above-mentioned Eq. (2) (4) simultaneous and consider them the "mathematical model for the starting process".

* Heat emissivity $\left(\mathrm{W} /{ }^{\circ} \mathrm{C}\right)=$ heat-transfer coefficient $\left(\mathrm{W} / \mathrm{m}^{2} \cdot{ }^{\circ} \mathrm{C}\right) \times$ area of heat-transfer surface $\left(\mathrm{m}^{2}\right)$.

\subsection{Lamp voltage and current wave- forms in the starting process}

To the starting method that has a switch in parallel with the lamp and leads the lamp to the operating state by switching on and off, the lamp voltage and current waveforms in the starting process were obtained by calculation.

The calculated results was compared with experimental ones and discussed.

In order to simplify calculation, the actual circuit (Fig. 1) is transcribed as Fig. 6, where switch is represented as resistance $R_{s}$.

Then $R_{s}=0$ means switching on and $R_{s}=\infty$ means switching off.

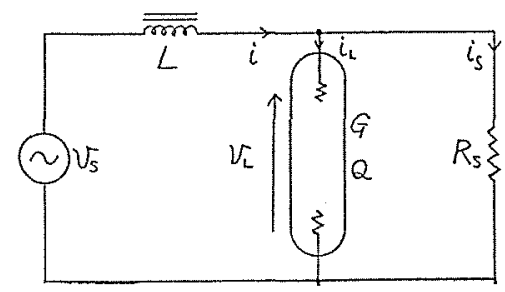

Fig. 6 Equivalent circuit.

Circuit equations :

$$
\begin{aligned}
& v_{s}=L \frac{d i}{d t}+v_{L} \\
& v_{L}=R_{s} \cdot i_{s} \quad \ldots
\end{aligned}
$$

$$
\begin{aligned}
v_{L} & =\frac{i_{L}}{G} \\
i & =i_{S}+i_{L} \\
p_{L} & =v_{L} \cdot i_{L}
\end{aligned}
$$

Mathematical model for the starting process:

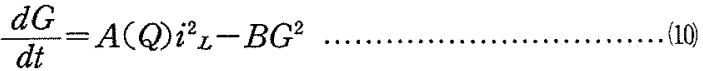

$$
\begin{aligned}
& A(Q)=A_{1}\left(1-\exp \left(-Q / A_{2}\right)\right) \ldots \ldots \ldots \ldots \ldots \ldots \ldots . . .(1)
\end{aligned}
$$

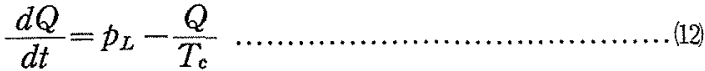

The above-mentioned simultaneous Eq. (5) (12) were calculated by Runge-Kutta method ${ }^{12)}$ using a digital electronic computer.

Where, $L$ is the inductance $(\mathrm{H})$ of a ballast, $v_{s}$ is the source voltage (V), $v_{L}$ is lamp voltage (V), $i$ is the current (A) flowing through the ballast, $i_{*}$ is the current (A) flowing through the switch, $i_{1}$ is the lamp current (A). In order to shorten the time of the operation, time axis was reduced for the calculation.

Figure 7 shows the flow chart of the calculation.

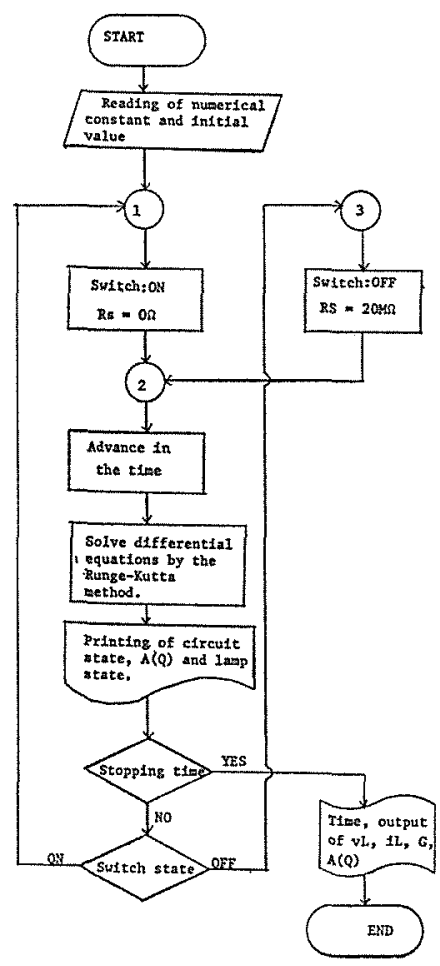

Fig. 7 Flow chart.

Figure 8 shows calculated results of the lamp voltage and current waveforms, and the value of $A(Q)$ as a function of time in the starting state.

The corresponding experimental results are shown in Fig. 9. The comparison between both figures shows that the results of the calculation by the authors reproduce the tendency of "the process in which the lamp is being fallen into the operating state, where the lamp voltage gradually decreases as the lamp current gradually increases." 


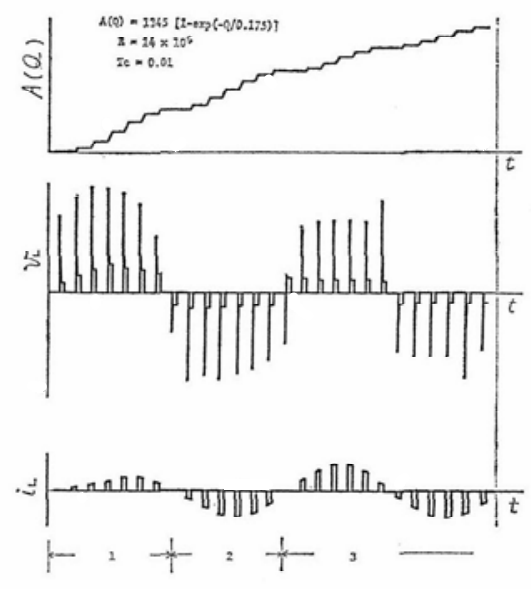

Fig. 8 Calculated results of the starting process.

1. Initial starting period.

2. Middle starting period.

3. Latter starting period.

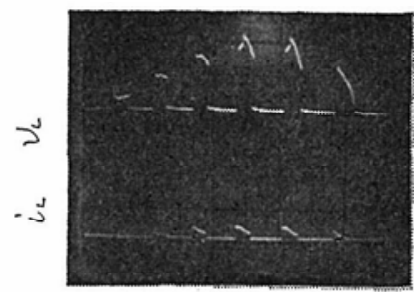

(a) 2

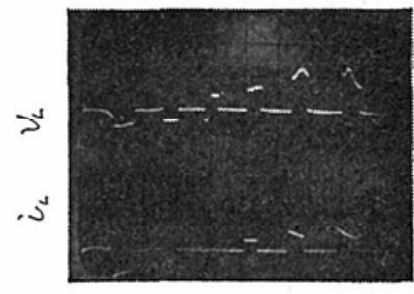

(b) 2

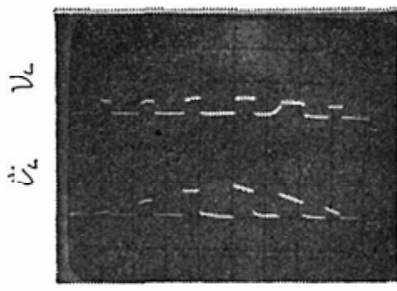

(c) 3

Fig. 9 Experimental results of the starting process.

1. Initial starting period.

2. Middle starting period.

3. Latter starting period.

4. Lamp voltage $(400 \mathrm{~V} / \mathrm{d})$.

5. Lamp current $(1 A / d)$.

As above-mentioned, the mathematical model shown in Eq. (2) (4) can represent the "process of transition from ignition to the operating state of discharge lamps" which cannot be represented by usual mathemaitcal models.

\section{Evaluation of whether or not the lamp can get to the operating state}

Consider the before-mentioned starting method for turning on and off the switch connected parallel to lamp.

Take up a $35 \mathrm{~W}$ low pressure sodium lamp as an example. And evaluate whether or not the lamp can get to the operating state to the combination of switch-on-off time. The comparison between the results calculatd by using the mathematical model for the starting process shown in section 2.1 and the actual experimental results is discussed.

\subsection{Semiconductor starting device for low pressure sodium lamp}

The starting method shown in Fig. 1 is explained as follows: A semiconductor starting device consisting of a diode bridge, switching transistor $\mathrm{Tr}$, an amplification circuit, an oscillation circuit and a detection circuit of the lamp voltage is connectd parallel with the lamp La. And lamp $\mathrm{La}$ is connected series with the source power Vs and a ballast Ch.

When the lamp is in the starting process and the lamp voltage is high, the detection circuit of the lamp voltage continues to supply output signals to the oscillation circuit.

The detection circuit of the lamp voltage acts to stop oscillation by holding the output signals when the lamp is fallen into the operating state and the lamp voltage is low.

When the lamp is in the starting process, the oscillation circuit acts to continue oscillation to control on-off of transistor. The amplification circuit amplifies the output from the oscillation circuit and supplies base current to the transistor. The transistor is synchronized to the amplified output from the amplification circuit and switches on and off both terminals of the lamp. The dioded bridge, independent of the polarities of the source power voltage, has the transistor switch on and off.

In short, when the lamp is in the starting process, the switch connected parallel to the lamp continues to go on and off. When the lamp is fallen into the operating state, the switch hold the offstate. On-time and off-time of the switch can be realized by changing the constant of the oscillation circuit.

When the switch is on, the short-circuit current flowing through the ballast is stored as magnetic energy in the ballast. When the switch is off, the short-circuit current is rapidly interrupted, and the stored magnetic energy is transformed into high pulse-voltage. It impacts on both terminals of the lamp. This high pulse-voltage increases the conductance of the lamp. When the switch is on again, the conductance of the lamp decreases during this period. When the switch is off, the above-mentioned operation increases the conductance of the lamp in the same manner. The conductance of the lamp 
gradually increases repeating the above-mentioned processes. Finally, the lamp is fallen into the operating state.

Therefore, the longer the on-period of the switch, the more the magnetic energy stored in the ballast increases. So, when the switch is turned off, the high pulse-voltage which impacts on both terminals of the lamp increases, and the increase of the conductance of the lamp is prompted. Thus, the lamp is enabled to be easily fallen into the operating state.

However, when the on-period is too long, compared with the off-period, the conductance of the lamp attenuates greatly, and it becomes more difficult for the lamp to be fallen into the operating state. The relationship between the combination of the on-time and off-time of the switch and the starting possibility (; whether or not the lamp can get to the operating state) is represented in the plan consisting of the on-time and off-time. The possible and impossible regions for the lamp to ignite can be seen.

\subsection{Evaluation of whether or not the lamp can get to the operating state}

The calculating procedure by using the mathematical model for the starting process consisting of $\mathrm{Eq}(2) \sim(4)$ is shown below.

First, turn on the lamp actually and obtain lamp voltage waveform and lamp current waveform in the operating state. Next, calculate the coefficients $A$ and $B$ in the Eq. (1) by using the methods of Ozaki, Masumi and Bou ${ }^{8)}$. Fix $B$, calculate $T$. in the Eq. (2) and $A_{1}, A_{2}$ in the Eq. (3) by method

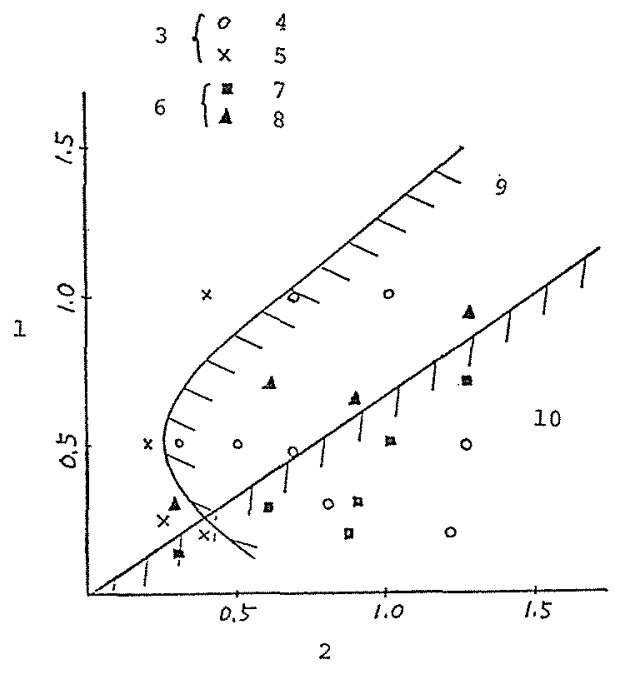

Fig. 10 Possible starting region of lamp.

1. Off-time. 2. On-time. 3. Calculation. 4. Starting possible 5. Starting impossible. 6. Experi. ment. 7. Starting possible 8. Starting impossible. 9. Possible. starting region (calculated result) 10. Possible startins region (experimental result) shown in section 2.1. In case of $35 \mathrm{~W}$ low pressure sodium lamp which the authors chose, the results are as follows: $A_{1}=1345\left(\mathrm{~S} /\left(\mathrm{s} \cdot A_{2}\right)\right), A_{2}=0.175$ (J), $\left.B=14 \times 10^{6}(1 / \mathrm{CS} \cdot \mathrm{s})\right), T_{r}=0.01(\mathrm{~s})$.

Substitute these numerical values for the Eq. $(10) \sim(12)$, and picture the lamp voltage, lamp current, conductance and $A(Q)$ as a function of time in the starting process by the method shown in section 2.2 (example Fig. 8).

For the combinations of various on-times and off-times, the calculations were carried out, and the starting possibility was evaluated from the increasing rate of $A(Q)$, the lamp current, and the conductance, and the decreasing rate of the lamp voltage in each figure. In order to shorten the time of the operation, the calculations were carried out only within 1.5 cycles.

The possible region obtained by the above-mentioned calculation is shown in the plan consisting of the on-time and off-time, which is Fig. 10. The possible starting region, obtained by experiments on the circuit shown in Fig. 1, is also shown in Fig. 10.

As shown in Fig. 10, the result was that the possible starting region by calculations was larger than that by experiments.

\section{Consideration}

As shown in section 2.2, the "mathematical model for the starting process introducing the thermal concept" can represent the process in which the lamp is fallen into the operating state. However, as shown in section 3.2 , on the evaluation of the starting possibility of the lamp, there is a difference between the calculated results and the experimental ones, and the calculated results tend to more greatly emphasize the starting possibility of the lamp, compared to experimental results.

The time constant $T_{c}$ of "the first-order lag heat quantity system "in" the mathematical model for the starting process" is identified in both the starting process and the operating state. Therefore, these results are considered to arise from the fact that the increasing ratio of the heat quantity in the starting process is too large. The below-mentioned are the problem yet to be solved to improve this point:

(a) Do not concentrate the effects of heat quantity $Q$ only on coefficient $A(Q)$. Assume that both coefficient $A$ and coefficient $B$ are function of heat quantity $Q$, and represents the starting process and the operating state, using the idential time constant $T_{c}$ of the heat quantity system. Concretly speaking, replace Eq. (4) in section 2.1 with the following one:

$$
\frac{d G}{d t}=A(Q) i^{2}-B(Q) G^{2}
$$

Each waveforms of the lamp conductance $G$, the time rate of change $d G / d t$ of conductance and lamp electric power $p_{L}$ are obtained from lamp voltage waveform $v_{L}$ and lamp current waveform $i_{L}$ in the operating state. 

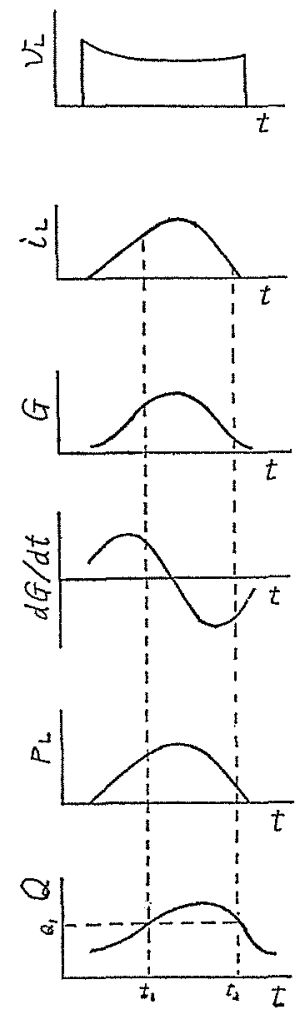

Fig. 11 Relationship between various electric quantities of lamp and heat quantity $Q$.

On the other hand, heat quantity $Q$ is obtained from the Eq. (2) by using the presumed time constant $T_{c}$. These relationships are shown in Fig. 11. In Fig. 11, at a certain time; $t_{1}$, there is a heat quantity; $Q\left(t_{1}\right)=Q_{1}$. As against it, there is an another time $t_{2}$. At $t_{2}, Q\left(t_{2}\right)=Q_{1}=Q\left(t_{2}\right)$ hold.

To each time of $t_{1}$ and $t_{2}$, the coefficients $A$ and $B$ each have identical value, i.e. $A\left(Q\left(t_{1}\right)\right)=A(Q$ $\left.\left(t_{2}\right)\right)=A_{1}, B\left(Q\left(t_{1}\right)\right)=B\left(Q\left(t_{2}\right)\right)=B_{1}$. However, to each time of $t_{1}$ and $t_{2}$, the lamp current $i_{2}$, the conductance $G$ and the time rate of change $d G / d t$ of the conductance have each different values. In other words, two different times, $t_{1}$ and $t_{2}$, exist to a certain heat quantity $Q_{1}$ and the following Eq. (14) and (15), are concluded.

$$
\begin{aligned}
& \left.\frac{d G}{d t}\right|_{t=t_{1}}=A_{1} i^{2}{ }_{L 1}-B_{1} G_{1}{ }^{2} \\
& \left.\frac{d G}{d t}\right|_{t=t_{2}}=A_{1} i^{2}{ }_{L 2}-B_{1} G_{2}{ }^{2}
\end{aligned}
$$

When they are solved, the coefficients, $A_{1}$ and $B_{1}$, to a certain heat quantity $Q_{1}$, are obtained. The coefficients, $A_{2}$ and $B_{2}$, to an another heat quantity $Q_{2}$ are obtained in the same manner. So are $A_{3}$ and $B_{3}$ to $Q_{3} \ldots \ldots$. By the above-menoioned method, the function forms $A(Q)$ and $B(Q)$ to the heat quantity $Q$ are obtained. Substitute these $A(Q)$ and $B(Q)$ for the Eq. (13). Calculate in the same way as in section 2.2 , and compare calculated results and experimental ones in the operating state. Consider whether or not the presumed time constant $T_{\mathrm{c}}$ is correct. If not, correct it. Repeat the above-mentioned processes and decide the time constant $T_{\mathrm{c}}$.

When it is decided, the function forms of the coefficients $A(Q)$ and $B(Q)$ become definite.

(b) Taking thermal radiation into consideration, heat emissivity $\alpha$ deciding the time constant $T_{0}$ (=heat capacity/heat emissivity) of the heat quantity system is the function of temperature. Generally, heat current $\Phi(W)$ is represented by the following Eq. (16), i.e.

$$
\Phi=h\left(\theta_{1}-\theta_{0}\right) S+\varepsilon \sigma\left(T_{1}^{4}-T_{0}^{4}\right) S .
$$

Where, $h$ is the heat transfer coefficient $\left(\mathrm{W} /\left(\mathrm{m}^{2}\right.\right.$. $\left.\left.{ }^{\circ} \mathrm{C}\right)\right), \varepsilon$ is the thermal radiation coefficient, $\sigma$ is Boltzmann's constant $\left(\mathrm{W} /\left(\mathrm{m}^{2} \cdot \mathrm{K}^{4}\right)\right) . S$ is the area of heat-transfer surface $\left(\mathrm{m}^{2}\right), \theta_{1}$, and $\theta_{0}$ are temperature $\left({ }^{\circ} \mathrm{C}\right)$, and $T_{1}$ and $T_{0}$ are absolute temperature $(\mathbf{K})$.

The Eq. (16) is transformed into the Eq. (17).

$$
\begin{aligned}
\Phi= & \left(h+\varepsilon \sigma\left(T_{1}^{3}+T_{1}{ }^{2} T_{0}+T_{1} T_{0}{ }^{2}+T_{0}{ }^{3}\right)\right) \\
& \times\left(\theta_{1}-\theta_{0}\right) S \ldots \ldots \ldots \ldots \ldots \ldots \ldots \ldots \ldots \ldots \ldots \ldots \ldots \ldots \ldots \ldots \ldots \ldots \ldots \ldots \ldots \ldots
\end{aligned}
$$

When the Eq. (17) is represented as the one (18), i.e.

$$
\Phi=K\left(\theta_{1}-\theta_{0}\right) S
$$

$K$ is called the coefficient of heat transfer $\left(W /\left(\mathrm{m}^{2}\right.\right.$. $\left.{ }^{\circ} \mathrm{C}\right)$ ). So, heat emissivity, $\alpha=K \cdot s$, becomes the function of temperature. The heat emissivity $\alpha$ rapidly increases as the temperature rises, i.e. as the heat quantity, $Q=C \cdot \theta$, increase. It is shown in Fig. 12.

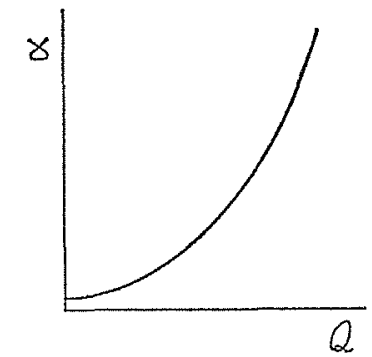

Fig. 12 Retationship between heat emissivity $\alpha$ and heat quantity $Q$.

Therefore, as the heat quantity $Q$ increases, the time constant $T_{c}$ decreases. This means that the time constant $T_{c}$ decrease when the lamp passing through starting process.

(c) The coefficient $A\left(Q_{1}\right)$ varies with the heat quantity $Q_{1}$ having large time constant $T_{o_{1}}$ and control the starting process. At the same time, the coefficient $B$ varies with another heat quantity $Q_{2}$ having small time constant $T_{\mathrm{c} 2}$, and becomes $B\left(Q_{2}\right)$ to control the operating state.

(d) It is not that the heat quantity $Q$ has a single time constant $T_{\mathrm{c}}$. The heat quantity system is the second-order one which has both of large time constant $T_{c_{1}}$ controlling the starting process and small time constant $T_{e_{2}}$ controlling the operat- 
ing state.

If those items are further investigated, it is considered that "the mathematical model precisely representing the whole processes from the starting process to the operating state" can be obtained.

\section{Conclusion}

The mathematical model which can represent the starting process were obtained by newly introducing a first-order lag heat quantity system into the usual mathematical model representing the operating state of discharge lamp. In this paper, the low pressure sodium lamp was chosen and discussed.

It is considered that the ideas of the mathematical model for the starting process introducing the thermal concept are applicable to other discharge lamps.

The problems yet to be solved for the mathematical model representing smooth transition from the starting process to the operating state and to precisely evaluate the starting possibility of the lamp are as follows:

(1) Examine (a) $\sim$ (d) shown in chapter 4 .

(2) Clarify the relationship with the physical phenomena in the lamp.
We, the authors, wish for this paper to be useful in advancing the analytic technology of discharge lamp.

\section{References}

(1) Francis, V. J., : Fundamentals of Discharge Tube Circuits (1948).

(2) Peek, S. C. Spencer, D. E. : Illum. Engng 63 (1968) 157166.

(3) Amano: Conf. on Inst. Elect. Engng. of Japan (1974) 149.

(4) Nishioka, Morimoto: Conference on Inst. Elect. Engng. of Japan (1970) 806.

(5) Masumi, Bou: J. Illum. Engng. Inst. of Japan 57 (1973) 341-348.

(6) Masumi, Bou: The Transactions of Inst. Elect. Engng. of Japan 50-A53 (1975) 409-415.

(7) Masumi, Bou : Conf. on Light Sources and Its Installations LS-77-24 (1977-11).

(8) Ozaki, Masumi, Bou: Conf. on Light Sources and Its Installations LS-78-11 (1978-9).

(9) Tanigawa, Wada: Matsushita Electric Works, Ltd. Technical report 16 (1978) 26-32.

(10) Wada, Tanigawa : Conf. on Illum. Engng. Inst. of Japan in Tokyo (1979) 21.

(11) Wada, Tanigawa: J. Illum. Engng. Inst. of Japan 65 (1981) $3 \sim 7$.

(12) Wylie, C. R.: Advanced Engineering Mathematics Upper volume (1975).

Recieved 31 May. 1982; Revision Received 16 Aug. 1982. 